\title{
Aromatic penta-linked hydrocarbons in soot nanoparticle formation
}

\author{
Laura Pascazio ${ }^{\mathrm{a}}$, Jacob W. Martin ${ }^{\mathrm{a}}$, Angiras Menon ${ }^{\mathrm{a}}$, Dingyu Hou ${ }^{\mathrm{b}, \mathrm{c}}$, \\ Xiaoqing You ${ }^{\mathrm{b}, \mathrm{c}}$, Markus Kraft ${ }^{\mathrm{a}, \mathrm{d}, *}$ \\ ${ }^{a}$ Department of Chemical Engineering and Biotechnology, University of Cambridge, West \\ Site, Philippa Fawcett Drive, Cambridge, CB3 0AS, United Kingdom \\ ${ }^{b}$ Center for Combustion Energy, Tsinghua University, Beijing, 100084, China \\ ${ }^{c}$ Key Laboratory for Thermal Science and \\ Power Engineering of Ministry of Education, Tsinghua University, Beijing, 100084, \\ China \\ ${ }^{d}$ School of Chemical and Biomedical Engineering, Nanyang Technological University, \\ Singapore 637459
}

\begin{abstract}
A new crosslinking reaction between two pentagonal rings around the periphery of planar pericondensed aromatic molecules is proposed and its impact on soot nanoparticle formation explored. The reaction mechanism was computed, using density functional theory, between an aryl-type $\sigma$-radical on a rim-based pentagonal ring attacking another rim-based pentagonal ring. A hydrogen migration allowed for the formation of a double bond forming a planar aromatic penta-linked hydrocarbon (APLH) complex, recently experimentally observed. The clustering of this planar species is compared with a pericondensed polyaromatic hydrocarbon $(\mathrm{PCAH})$ and an aromatic aryllinked hydrocarbon (AALH) using molecular dynamics and metadynamics. Similar clustering is found for the investigated species compared with a peri-
\end{abstract}

\footnotetext{
*Corresponding author

Email address: mk306@cam.ac.uk (Markus Kraft)
} 
condensed structure of similar mass indicating enhanced physical interactions after forming the crosslink. Finally, a further crosslink is possible between the unsaturated pentagonal ring sites forming an aromatic rim-linked hydrocarbon (ARLH) complex of considerable stability. This was confirmed by simulating the stable molecular dynamics of such a complex with on-the-fly quantum forces from a quantum semi-empirical method, revealing possible reactions under flame conditions that might play a role in soot nucleation. Keywords:

soot formation, molecular dynamics, localized $\pi$-radical, aromatic rim-bonded hydrocarbons, aromatic penta-linked hydrocarbons

*Supplementary Material is available for this paper.

Colloquium submitted for: Soot, Nanomaterials, and Large Molecules

Method of Determination: Method 2

Total Word Count: 4163 words, 8 pages 


\section{Introduction}

Carbon particulate pollution leads to respiratory diseases, contributes to global warming while in the atmosphere and leads to increased sea ice loss through reduced albedo [1-3]. Much research has been focused on understanding the formation mechanism of soot in order to reduce the amount produced by human activities. The critical stage of particulate formation, which remains the least well understood, is the inception of gas phase aromatics to form condensed clusters of soot nanoparticles [4].

Mass spectrometry indicates that pericondensed polyaromatic hydrocarbons $(\mathrm{PAH})$ are the primary species in flames and the first suggested explanation of rapid soot formation was physical aggregation of these large aromatics through dispersion interactions. However, ab initio and molecular dynamics (MD) calculations have indicated that for the size of aromatics experimentally found in flames (up to approximately 15 rings [5]), the intermolecular forces are insufficient for them to cluster at flame temperatures where soot forms [6].

Chemical inception mechanisms involving reactive aromatics have also been thought to contribute to soot inception [4]. Mass spectrometry has indicated that crosslinking reactions form larger PAH in sooting flames. Studying the formation of soot and fullerenes in low pressure flames using mass spectrometry [7], found evidence for a crosslink reaction forming aromatic oligomers or aromers. More recent mass spectrometry has confirmed the presence of a crosslinked species (30-50 carbon atoms) containing two smaller aromatic fragments (10-20 carbon atoms) with hydrogen to carbon ratios indicating pericondensed species [8]. This formation of aromers was found to 
correspond to the transition between a non-sooting and sooting flame in a low pressure methane burner, indicating that the fragments contained in the given aromer were present in the flame and that aromer formation might be an important reaction in soot formation.

Crosslinking of PAH aryl-type $\sigma$-radicals on hexagonal rings with other PAH has previously been considered $[9,10]$. Crosslinking requires the abstraction of a hydrogen atom to form the $\sigma$-radical. In most schemes, such as with aromatic aliphatically/aryl-linked hydrocarbons (AALH), a second hydrogen abstraction is required to form the stable species, which leaves the initial crosslink open to thermal fragmentation. This AALH crosslink with a hexagonal ring leads to steric interactions with the neighboring terminating hydrogen atoms, twisting the two aromatic planes of the monomers relative to each other and resulting in a non-planar crosslinked molecule. Metadynamics analysis has shown that this steric effect significantly reduces the intermolecular interactions [11]. This is due to the short range of the dispersion interactions (scaling as $r^{-6}$ ) that are easily disrupted by thermal or steric effects. Therefore, planar species are optimal for clustering due to the short range nature of dispersion interactions.

Coupling mass spectrometry with photoionisation from a tunable XUV synchrotron source has lead to a reevaluation of isomeric species present in the flame. The mass ion $202 \mathrm{u}$, for example, is often matched to the pyrene species. However, the presence of pentagon-containing fluoranthene is also required to provide the photoionisation curve for this ion [12]. Mass spectrometry also suggests a slightly higher carbon to hydrogen ratio than anticipated from $D_{6 h}$ PAH (PAH having six-point symmetry) indicating that pentagonal 
rings are present on the rim [8]. High resolution atomic force microscopy (HR-AFM) was able to directly image some PAH found in nearly-sooting flames and confirm the presence of these rim-based pentagonal rings [13]. The authors in [13] also imaged fully- and partially- saturated rim-based pentagonal rings as well as planar crosslinked species containing a double bond between two pentagonal rings (inset of Fig. 1). We will call the latter an aromatic penta-linked hydrocarbons (APLH). APLH molecules are a special case of an AALH where saturation can form a double bond, so the two molecular planes are coplanar. This planarity might allow for more effective van der Waals clustering than the crosslinking between hexagonal rings [13]. We also recently found that the partially saturated site forms a localized $\pi$-radical of considerable reactivity using electronic structure calculations and reactivity indices [14]. This $\pi$-radical site, localized to the edge of the molecule, was found to be capable of bonding and stacking into aromatic rim-linked hydrocarbons (ARLH) indicating a potentially important role in soot formation [14].

The aim of this paper is to consider crosslinking reactions between two smaller aromatics that can lead to a planar molecule. A new crosslinking reaction between two pentagonal rings around the periphery of a PAH is proposed using hybrid density functional theory. The resulting product is a localized $\pi$-radical species similar to that found in [13]. The role of these radical species that are capable of bonding and stacking is investigated using density functional theory and quantum molecular dynamics (QMD) and the physical dimerization of the saturated aromatic product is explored using well-tempered metadynamics and MD simulations. 


\section{Reaction mechanism}

Processes involving PAHs containing pentagonal rings have been studied and investigated in the context of PAH growth. These include fivemember ring desorption, migration, rearrangement, bay closure and capping [15-17]. We have studied the reactivity of rim-based pentagonal rings towards crosslinking extensively in our previous study [14]. In this section we explored possible formation mechanisms of a crosslinked planar aromatic molecule with a double bond connecting two pentagonal rings on the rim of the two aromatic subunits for the first time.

Figure 1 presents the potential energy diagram for the investigated formation mechanism. All the molecular structures of stable chemical species and transition states involved in the reactions were optimized and the single point energies were calculated using the dispersion corrected hybrid density functional M06-2X-D3/cc-pVTZ. The method has been previously benchmarked and found to provide accurate values (errors of $<1 \mathrm{kcal} / \mathrm{mol}$ for hydrogen abstractions [18] and $<2 \mathrm{kcal} / \mathrm{mol}$ for hydrocarbons [19]). The Gaussian 16 software [20] was used to perform the geometry optimisations to determine minimum energy and transition states. Frequencies were also determined to ensure transition states possess a single imaginary frequency corresponding to the reaction.

The reaction mechanism starts with two acenaphthalene molecules (1a in Fig. 1). First, hydrogen abstraction by an $\mathrm{H}$ radical occurs, forming a $\sigma$-radical $\mathbf{1 b}$ on one of the acenaphthalene pentagonal rings. This is known to be the predominant reaction mechanism for PAH growth [21-23]. Rimbased pentagonal rings in PAH are non-aromatic cycles. This results in 
strong electron localisation around the rim, and hence a very reactive site where hydrogen addition reactions can also occur (as opposed to just abstraction reactions), in which case a localised $\pi$-radical is formed. Low barriers are seen for hydrogen addition compared with hydrogen abstraction (see supplementary information). The abstraction reaction from a rim-based pentagonal ring has an energy barrier of $18.8 \mathrm{kcal} / \mathrm{mol}$, slightly higher than the energy barrier for abstraction from the hexagonal ring. This difference of $2.6 \mathrm{kcal} / \mathrm{mol}$ (see supplementary information) in barrier height can reduce the likelihood of H-abstraction from the pentagonal ring compared to the hexagonal ring at low temperatures, though the effect would be less evident at high temperatures [24].

In our previous publication we explored the set of possible crosslinks between reactive edges that are thermally stable at soot forming temperatures [14]. We can exclude aromatic $\sigma$-radical recombination due to its low

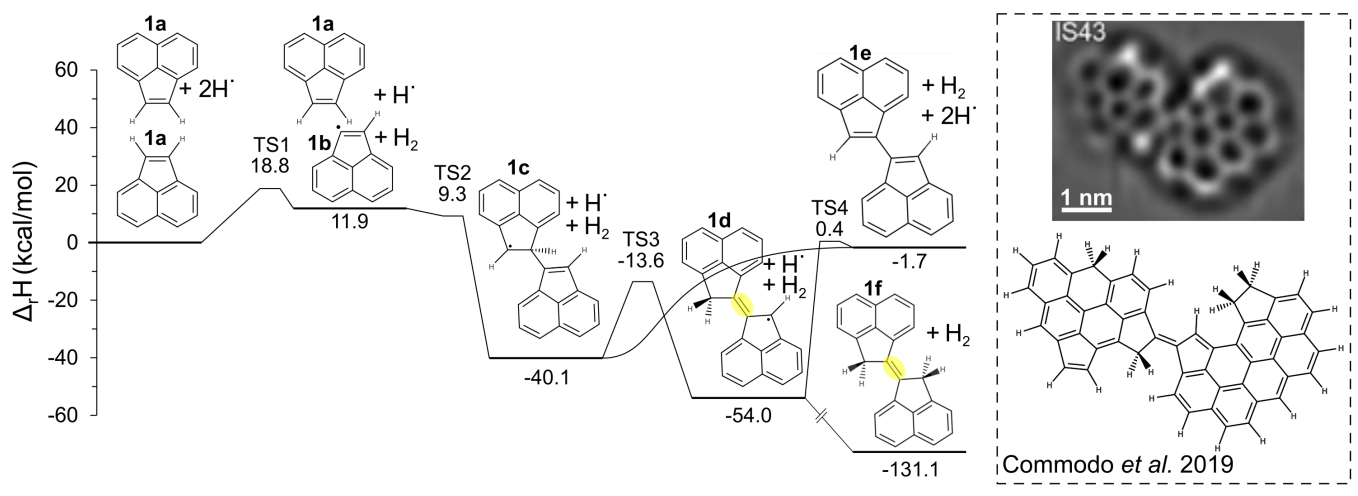

Figure 1: Potential energy diagram for the formation of a planar crosslinked aromatic molecule with a double bond (highlighted) connecting rim-based pentagonal rings of the aromatic subunits at $0 \mathrm{~K}$. A similar species observed experimentally has been also reported for comparison [13] used with permission CC BY-NC-ND 4.0. 
collision efficiency [25]. Localised $\pi$-radicals form only weak bonds with rimbased pentagonal rings, while the $\sigma$-radical $\mathbf{1 b}$ attacking the rim-based pentagonal ring 1a forms a stable crosslink that can undergo further reactions. A significantly higher dissociation barrier of $49.4 \mathrm{kcal} / \mathrm{mol}$ is found for the reaction of $\mathbf{1 b}$ with a rim-based pentagonal ring compared with phenanthrene or naphthalene (the bond energies are $\approx-36$ and $\approx-38 \mathrm{kcal} / \mathrm{mol}$ respectively), further indicating the special reactivity of this non-aromatic ring. The transition state for forming 1c has a submerged energy barrier of $-2.6 \mathrm{kcal} / \mathrm{mol}$ due to it forming a van der Waals complex. From here the hydrogen can migrate to the neighbouring site. This forms a highly stable planar complex, the aromatic penta-linked hydrocarbon (APLH) 1d, without requiring a subsequent hydrogen abstraction. Another possible pathway involves direct hydrogen loss from 1c forming the completely unsaturated product 1e. This geometry is not an APLH as it does not possess the double bond crosslink and the aromatic planes are not coplanar. The energy needed for $\mathrm{H}$ directly lost from $1 \mathrm{c}$ is $38.4 \mathrm{kcal} / \mathrm{mol}$ (no TS was found) indicating that the $\mathrm{H}$ migration (energy barrier of $26.5 \mathrm{kcal} / \mathrm{mol}$ ) is favoured. It should also be noted that hydrogen addition to $\mathbf{1 e}$ will produce the $\mathbf{1 d}$ APLH species, with further addition giving 1f. Hydrogen abstraction can also remove hydrogen from these saturated species. This suggests a partial equilibrium of $\mathbf{1 d}, \mathbf{1 e}$ and $\mathbf{1 f}$ with $\mathrm{H} / \mathrm{H}_{2}$ in the flame that we have seen for acenapthalyene [26]. The high energy needed to have $\mathrm{H}$ directly lost from $1 \mathbf{d}$ to form $1 \mathrm{e}(54.4 \mathrm{kcal} / \mathrm{mol})$ suggests that this pathway is not competitive.

There is a potential chain reaction that could occur if the structure contains two or more pentagonal rings. Subsequent hydrogen abstraction on an- 
other rim-based pentagonal ring would form another $\sigma$-radical able to attack a third PAH, however, given the barrier for hydrogen abstraction we doubt such a mechanism would provide a significant nucleation flux. However, like the reaction with resonantly stabilized radicals, it would only require a single hydrogen abstraction [27].

Another interesting aspect of species $\mathbf{1 d}$ is that it has a partially saturated rim-based pentagonal ring. Therefore, it forms a localized $\pi$-radical species as we have previously shown [14]. This species can then follow two routes, as detailed below: 1) the radical site can be protonated forming a closed shell species (1f) that cannot chemically dimerize but may physically dimerize. However, we expect that $\mathbf{1 f}$ is too small to physically dimerize at flame temperatures [6] so we investigated the physical dimerization for a protonated APLH of bigger size in the following paragraph. 2) it can stay in its $\pi$-radical form $\mathbf{1 d}$ and chemically dimerize with another localized $\pi$-radical.

\section{Physical dimerization of aromatic penta-linked hydrocarbons}

The physical dimerization of the protonated closed-shell APLH discussed above was explored using well-tempered metadynamics and MD simulations. These two techniques give different information about the process. Welltempered metadynamics is able to determine the thermodynamic stability of atomic and molecular systems and maps the free energy surfaces of the system considered, providing the minimum free energy configurations and the energy barriers between them. The dimerization process is investigated using this technique. However, metadynamics is not able to capture the behavior of a large system of interacting molecules. To study this, molecular dynamics 
calculations were run to provide some time-dependent quantities such as the size of nucleated clusters, cluster lifetimes and coagulation efficiencies at different conditions.

The clustering behaviour of a protonated APLH containing two $\mathrm{C}_{18} \mathrm{H}_{10}$ subunits, $\mathbf{2 a}$, is compared to two other molecules of similar sizes but different structure. In particular, $2 \mathbf{b}$ also contains two $\mathrm{C}_{18} \mathrm{H}_{10}$ subunits but with an AALH type crosslink between two hexagonal rings, and $\mathbf{2 c}$ is a pericondensed aromatic hydrocarbon $(\mathrm{PCAH})$ obtained from the ring closure of the crosslinked species $\mathbf{2 b}$. The latter two kinds of molecules have previously been considered as precursor for soot nucleation [11]. This work focuses on different types of PAHs that we believe are currently the most interesting for the community. There are already studies of the clustering behaviour as function of size $[6,11,28]$, so only one size is selected here. The molecular mass of $\approx 500 \mathrm{u}$ was chosen, as clustering with this periodicity has been observed in mass spectrometry studies [29]. The structures are given in Fig 2.

The OPLS-AA forcefield was used to model the intramolecular interactions [30] and the iso-PAHAP force field [6] was used to model intermolecular interactions. The atomistic electrostatic potential charges were obtained from electronic structure calculations using the Gaussian 16 [20] program at the B3LYP/cc-pVTZ level of theory. Atom-atom non-bonded interactions were cut off at $4 \mathrm{~nm}$.

\subsection{Free energy profiles}

The free energy (FE) calculation of the physical dimerization of these selected aromatic molecules was performed using the GROMACS 5.1 software [31] coupled with the PLUMED plugin [32] at three different temperatures $(500,1000$, and $1500 \mathrm{~K}) .1500 \mathrm{~K}$ is used as upper limit of the temper- 


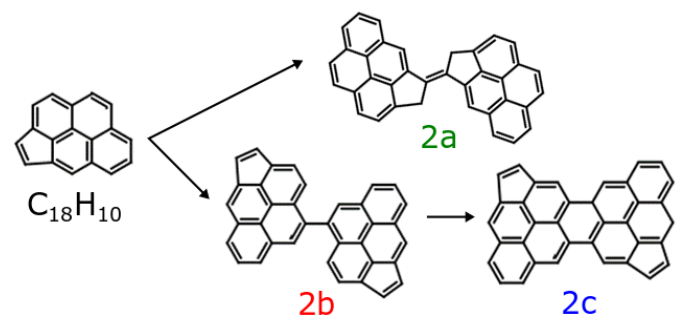

Figure 2: Molecules considered for molecular dynamics and metadynamics simulations: a planar APLH (2a), an AALH (2b) and a PCAH (2c).

ature range because it is the temperature around which soot seems to begin to nucleate. Molecules were first minimized before starting well-tempered metadynamics simulations. Temperatures are kept constant by employing a Langevin thermostat with a time constant of 0.1 ps. Each FE profile is built as the average of three independent $100 \mathrm{~ns}$ runs, in which the Gaussianshaped bias was deposited every 100 fs. The Gaussian-shaped bias had an initial width and height of $0.04 \mathrm{~nm}$ and $0.1 \mathrm{kcal} / \mathrm{mol}$ respectively, with bias factor set to 10. For this process, the collective variable chosen to represent whether the system is in a monomer state or dimer state is the distance between the center of masses (COM) of the two monomers. An upper wall is placed at COM distance of $4.5 \mathrm{~nm}$ which limits the region of the phase space accessible during the simulation. The dimerization propensity is computed as the $\mathrm{FE}$ difference $(\triangle F E)$ between the dimer-state (minimum of the $\mathrm{FE}$ ) and the monomer-state (FE calculated at long distances, specifically when the molecular COM distance is above $3.8 \mathrm{~nm}$ ) according to Elvati et al. [11]. Figure 3 reports the computed free energies of homodimerization of molecules $\mathbf{2 a}, \mathbf{2} \mathbf{b}$, and $\mathbf{2 c}$ as function of the distance between centers of mass of the monomers at $500 \mathrm{~K}, 1000 \mathrm{~K}$, and $1500 \mathrm{~K}$. 


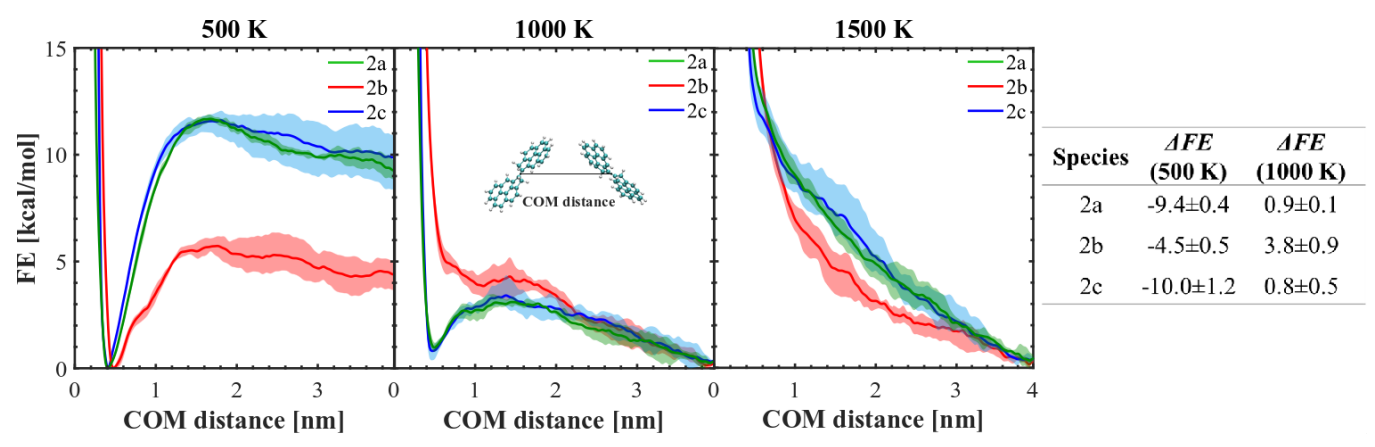

Figure 3: Free energies $(F E)$ profiles of homodimerization of species $\mathbf{2 a}, \mathbf{2} \mathbf{b}$, and $\mathbf{2} \mathbf{c}$ as function of the distance between centers of mass of the monomers (COM distance) at $500 \mathrm{~K}, 1000 \mathrm{~K}$ and $1500 \mathrm{~K}$. Variation between runs are indicated by the shaded areas. Dimerization propensity $(\triangle F E)$ of species $\mathbf{2 a}, \mathbf{2} \mathbf{b}$, and $\mathbf{2} \mathbf{c}$ at different temperatures are reported in $\mathrm{kcal} / \mathrm{mol}$ in the table on the right. No values were found for $1500 \mathrm{~K}$.

The FE profiles for all the investigated compounds do not show any dimerization propensity at $1500 \mathrm{~K}$ (no local minimum found in the FE profile) indicating that the entropic term dominates, in accordance with previous results reported in literature for molecules of similar sizes [28]. The dimer-state is found to be favored over the monomer-state only at $500 \mathrm{~K}$ for all the investigated molecules. In the temperature range $500 \mathrm{~K}-1000 \mathrm{~K} \mathbf{2 a}$ and $\mathbf{2 c}$ bind more strongly than $\mathbf{2} \mathbf{b}$ despite all three being of similar sizes. This behaviour is likely related to the planarity of the molecules and it suggests that the presence of the $\sigma$-bond in $\mathbf{2} \mathbf{b}$ destabilizes the dimer because the two aromatic sub-units are able to rotate with respect to each other around the $\sigma$ aliphatic bond. This is in agreement with previous results reported for molecules that contain an aliphatic/aryl-bond [11]. To explore how coplanarity effects the results we added a restraint to hold the rotation of the $\sigma$-bond in $\mathbf{2 b}$ to keep the whole molecule near planar (it cannot be fully planar due to the $\mathrm{H}$ atom 
steric effects). This gave similar results of $\Delta \mathrm{FE}$ for $\mathbf{2} \mathbf{b}, \mathbf{2} \mathbf{a}$ and $\mathbf{2} \mathbf{c}$ confirming that the coplanarity is critical (Fig. S3 in the supplementary information). Therefore the $\mathbf{2} \mathbf{a}$ and $\mathbf{2} \mathbf{c}$ profiles are quite similar due to both being planar species that can maximize dispersion interactions.

\subsection{Nucleating behaviour}

The dynamic behaviour of homomolecular systems for the three PAHs reported above was analysed using MD simulations in the canonical NVT ensemble in order to observe their clustering behaviour. Each simulation contained 500 molecules at a concentration of $1 \cdot 10^{18} \mathrm{~cm}^{-3}$ as used in Ref. $[6,33]$. A chain of ten Nosé-Hoover thermostats and a time constant of 100 fs were used to maintain a constant temperature. Three system temperatures were studied with the highest temperature corresponding to a typical flame temperature: $500 \mathrm{~K}, 1000 \mathrm{~K}$, and $1500 \mathrm{~K}$. Molecules were initially randomly located and orientated in a cube with periodic boundary conditions and initial velocities generated from a Maxwell-Boltzmann distribution at the given temperature. The integration time step was set to $1 \mathrm{fs}$ and trajectories were integrated using the velocity Verlet algorithm. All simulations were performed using the GROMACS 5.1 software [31]. Three trajectories of $1 \mathrm{~ns}$ each were run for each system and the clustering data was averaged over these runs.

For identifying a cluster, we defined a cut-off distance $\left(r_{\text {crit }}=1.2 \mathrm{~nm}\right)$ between two molecules' centers of mass and a critical period of time $\left(t_{\text {crit }}=20 \mathrm{ps}\right)$ used in previous studies [6].

Figure 4a shows the averaged percentage of bound molecules as a function of time for each species at each temperature. As temperature increases 

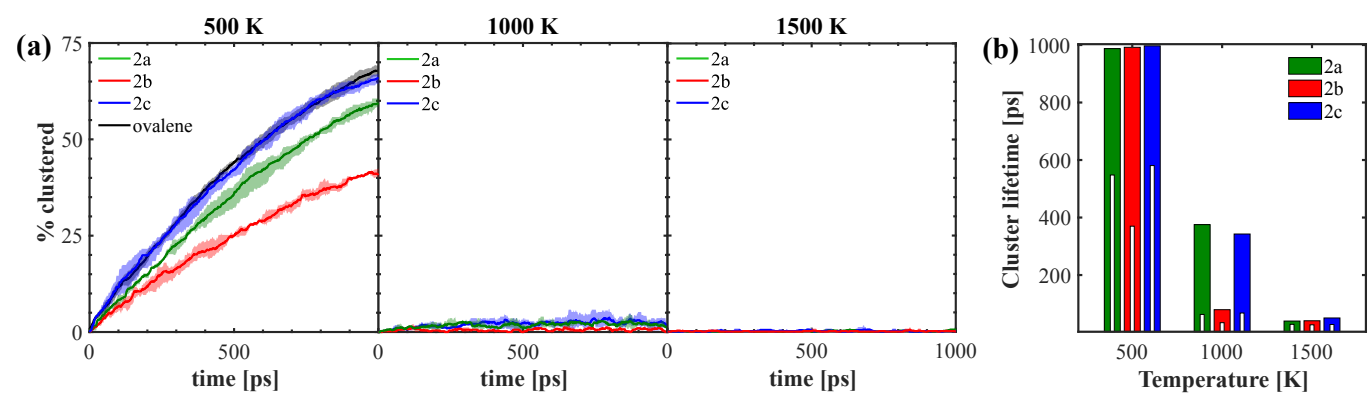

Figure 4: (a) Percentage of molecules in a bound state for species $\mathbf{2 a}, \mathbf{2} \mathbf{b}$ and $\mathbf{2} \mathbf{c}$ as function of the simulation time at 500, 1000 and $1500 \mathrm{~K}$. Results for the ovalene case at $500 \mathrm{~K}$ taken from Ref. [6] are also reported for comparison. Variations between runs are indicated by the shaded areas. (b) Maximum lifetimes (averaged lifetimes shown in white bars) at different temperatures for species $\mathbf{2 a}, \mathbf{2} \mathbf{b}$ and $\mathbf{2 c}$.

the expected decrease is observed in the percentage of molecules bound. The maximum cluster size is also seen to reduce for all molecules (see supplementary information). At temperatures higher than $1000 \mathrm{~K}$, no significant clustering is observed, as only relatively small clusters (dimers) are formed. At $500 \mathrm{~K}$ much larger clusters are formed for all the systems. At 500 and $1000 \mathrm{~K}$ there is little difference between the clustering statistics for the two planar molecules, $\mathbf{2} \mathbf{a}$ and $\mathbf{2} \mathbf{c}$, whereas the proportion of bound molecules is substantially less for $\mathbf{2} \mathbf{b}$, as expected from the results in Fig. 3. In Fig. 4a the results for a similar size PAH molecule (ovalene) at $500 \mathrm{~K}$ taken from Ref. [6] are also reported for comparison. The similar results obtained for the ovalene case and $\mathbf{2 c}$ molecule thus confirm that the clustering statistics for planar PAHs are mainly dependent on the number of aromatic rings rather than their shape. A similar trend is found for cluster lifetimes (Fig. 4b). At low temperatures cluster lifetimes are generally longer and some clusters survived the whole period of simulation for all the three species. At $500 \mathrm{~K}$, 
species 2a and 2c have an average cluster lifetimes of 548 and 581 ps, respectively, whereas species $\mathbf{2} \mathbf{b}$ has a much lower average lifetime of 370 ps. As the temperature increases, the maximum and average lifetimes of clusters both tend to decrease for all three species. The difference between the average lifetimes of clusters for the planar $\mathbf{2 a}$ and $\mathbf{2} \mathbf{c}$ and non-planar species $\mathbf{2 b}$ decreases with increasing temperature up to $1500 \mathrm{~K}$, where they all have similar values $(\approx 28 \mathrm{ps})$.

We expect that the physical interaction will not change between the saturated species and its localized $\pi$-radical form and that the results derived from the saturated molecules can be applied to the localized $\pi$-radical species. Due to the similar results that we obtained for the APLH and PCAH molecules, the thermodynamic stability and the clustering behaviour of APLH would be similar to a PCAH of the same size [6], indicating that only compound as large as circumcoronene can produce dimers that survive for more than $50 \mathrm{ps}$ under flames conditions. The concentration of such large PAHs is too low to make them drive nucleation [4]. At the same time, if smaller molecules than the one we studied here are considered, we expect that the average cluster lifetimes wouldn't change considerably in the size range from hexabenzocoronene to pyrene as reported in [6] due to the similarity between APLH and PCAH behaviour. In the absence of growth or chemical bond formation, the PCAH and APLH dimer lifetimes are around $\approx 20-30$ ps at $1500 \mathrm{~K}$ for species containing 4-10 rings. This implies that in absence of other processes, the physical dispersion forces are insufficient to stably bind clusters of aromatic species at flame temperatures, suggesting that other processes are needed. 


\section{Chemical dimerization of aromatic penta-linked hydrocarbon lo- calized $\pi$-radicals}

While the physical interaction appears to be unable to justify soot nucleation, the localized $\pi$-radical can form a chemical bond after physical dimerization. These localized $\pi$-radicals on rim-based pentagonal rings were recently found to form strongly bound stacked complexes, indicating a poten-

tially important role in soot formation [14]. In this section, we aim to explore the crosslinking between two $\mathbf{1 d}$ species and the formation of an ARLH complex. The stability of the crosslinks formed is systematically determined by calculating the binding energies using the dispersion corrected hybrid density functional M06-2X-D3/cc-pVTZ as reported in Ref. [14]. It should also be mentioned that we found that this level of theory systematically overbinds the dispersion interactions in PAH by $6 \pm 1 \mathrm{kcal} / \mathrm{mol}$ compared with SAPT(DFT) reference calculations. The binding energies of both purely physical APLH dimers and the cis and trans configurations of the chemically bound ARLH complex are reported in Fig. 5. The chemical bond in the ARLH increases considerably the binding energy between the monomers (Fig. 5a and b) compared to the physical interactions (Fig. 5c and d), 39.4 and $44 \mathrm{kcal} / \mathrm{mol}$ for the cis and trans complexes, respectively. Due to the high reactivity of radical species, the lifetimes of the physical dimers found in the previous section at $1500 \mathrm{~K}(\approx 28 \mathrm{ps})$ might be long enough to ensure the formation of an ARLH complex. This chemical bond could then provide the stability necessary for subsequent evolution into soot nanoparticles via another activated localized $\pi$-radical.

QMD simulations are computed with on-the-fly quantum forces us- 
a)

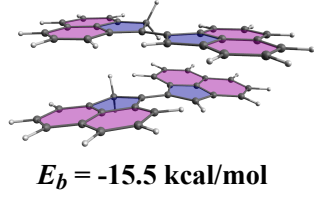

c)

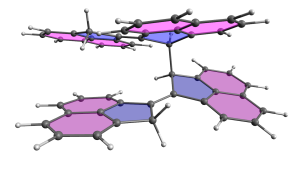

$E_{b}=-54.9 \mathrm{kcal} / \mathrm{mol}$

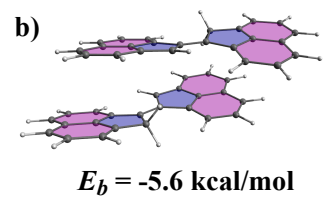

d)

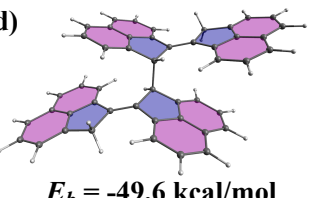

$E_{b}=-49.6 \mathrm{kcal} / \mathrm{mol}$

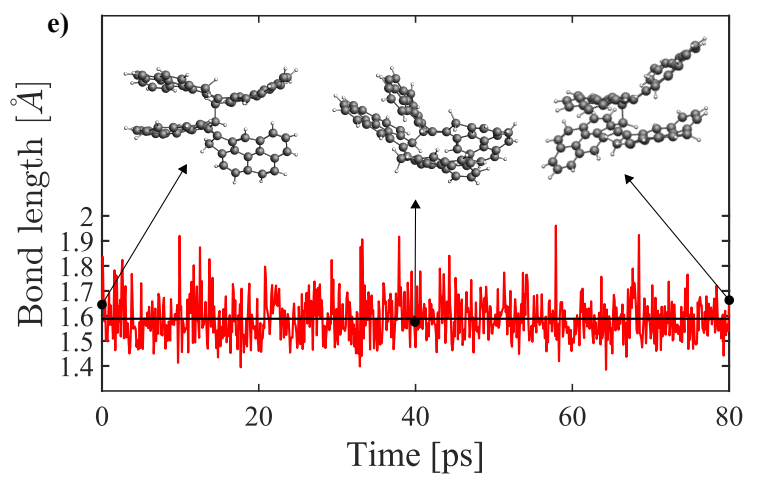

Figure 5: a) - d) Structures with calculated binding energies M06-2X-D3/cc-pVTZ. e) Snapshots of the QMD of species c).

ing AMS2019-MOPAC software [34] to confirm the high stability of the bonded complex. This method allows us to examine, in a more realistic manner, the dynamics of the chemical dimer at high temperatures. The semi-empirical parametric method 7 (PM7) Hamiltonian [35] was used as the interatomic manybody potential, which includes an empirical dispersion correction. Semi-empirical methods are quantum mechanical in nature, and thus preferable to classical forcefields because they represent atomic interaction forces more realistically allowing bond breaking and forming. At each MD simulation time step, self-consistent-field convergence of PM7 wavefunctions was achieved. A velocity Verlet integrator is used with a time step of 
0.5 fs. The molecule was firstly equilibrated at a temperature of $1500 \mathrm{~K}$ with a 5 ps MD run, during which the temperature was kept constant using a Nosé-Hoover thermostat. After equilibration, the simulation was conducted at $1500 \mathrm{~K}$ for $80 \mathrm{ps}$ of simulation. The results of the simulation are reported in Fig. 5e. The ARLH is stable throughout the 80 ps with a mean value of the length of the bond between APLH molecules centered at $1.589 \AA$. The bond length was found to fluctuate up to a maximum of $\approx 2 \AA$ corresponding to upper energy of $22 \mathrm{kcal} / \mathrm{mol}$. This is below the value of the bond dissociation computed at the higher level of theory, M06-2X-D3 (see supplementary information). This shows that over the length of the simulation timescale 80 ps we do not expect the bond to break. However, over longer time higher energy variation could break the bond. This simulation suggests a significant stability of the ARLH molecules under flame condition compared with

physical interactions. However, further investigation are required to explore whether the physical dimer lifetime is sufficiently long to allow chemical bond formation to occur and will be the subject of future work.

\section{Conclusion}

In this work, a new crosslinking reaction mechanism between pentagonal rings on the rim of planar pericondensed PAH is proposed. The physical and chemical dimerization of the resulting planar APLH is investigated using different modeling techniques. The APLH has a similar clustering behaviour to a planar pericondensed PAH of equivalent mass indicating enhanced physical interactions after forming the double-bond crosslink, however insufficient clustering was found at flame temperatures. While the physical interaction appears to be unable to justify soot nucleation (it cannot stabilize the 
dimer under flames condition giving dimers with average lifetimes of 30 ps), the chemical bond formed between the two APLH radical unsaturated pentagonal ring sites gives an ARLH forming a $\pi$-stacked and covalently bonded complex of considerable stability under flames condition. This was confirmed by simulating the molecular dynamics of such a complex with quantum on-the-fly forces revealing the potential of novel crosslinking reactions and penta/rim-linked aromatics in soot formation.

\section{Acknowledgments}

This project is supported by the National Research Foundation (NRF), Prime Minister's Office, Singapore under its Campus for Research Excellence and Technological Enterprise (CREATE) programme. This project has received funding from the European Union's Horizon 2020 Research and Innovation Programme under grant agreement no. 724145. The authors are grateful to EPSRC (grant number: EP/R029369/1) and ARCHER for financial and computational support as a part of their funding to the UK Consortium on Turbulent Reacting Flows (www.ukctrf.com).

\section{References}

[1] P. J. Landrigan, R. Fuller, N. J. R. Acosta, O. Adeyi, et al., The Lancet Commission on pollution and health, The Lancet (2017).

[2] J. R. McConnell, R. Edwards, G. L. Kok, M. G. Flanner, C. S. Zender, E. S. Saltzman, J. R. Banta, D. R. Pasteris, M. M. Carter, J. D. W. Kahl, 20th-Century Industrial Black Carbon Emissions Altered Arctic Climate Forcing, Science 317 (2007) 1381-1384. 
[3] T. C. Bond, S. J. Doherty, D. W. Fahey, P. M. Forster, T. Berntsen, et al., Bounding the role of black carbon in the climate system: A scientific assessment, J. Geophys. Res.:Atmospheres 118 (2013) 53805552.

[4] H. Wang, Formation of nascent soot and other condensed-phase materials in flames, Proc. Combust. Instit. 33 (2011) $41-67$.

[5] M. L. Botero, E. M. Adkins, S. González-Calera, H. Miller, M. Kraft, PAH structure analysis of soot in a non-premixed flame using highresolution transmission electron microscopy and optical band gap analysis, Combust. Flame 164 (2016) 250-258.

[6] T. S. Totton, A. J. Misquitta, M. Kraft, A quantitative study of the clustering of polycyclic aromatic hydrocarbons at high temperatures., Phys. Chem. Chem. Phys 14 (2012) 4081-94.

[7] K.-H. Homann, Fullerenes and soot formation - new pathways to large particles in flames, Angew. Chem. Int. Ed. 37 (1998) 2434-2451.

[8] P. Desgroux, A. Faccinetto, X. Mercier, T. Mouton, D. A. Karkar, A. El Bakali, Comparative study of the soot formation process in a "nucleation" and a "sooting" low pressure premixed methane flame, Combust. Flame 184 (2017) 153-166.

[9] A. Violi, A. Kubota, T. Truong, W. Pitz, C. Westbrook, A. Sarofim, A fully integrated kinetic monte carlo/molecular dynamics approach for the simulation of soot precursor growth, Proc. Combust. Instit. 29 (2002) $2343-2349$. 
[10] A. Violi, A. F. Sarofim, G. A. Voth, Kinetic Monte Carlo-molecular dynamics approach to model soot inception, Combust. Sci. Technol. 176 (2004) 991-1005.

[11] P. Elvati, K. Turrentine, A. Violi, The role of molecular properties on the dimerization of aromatic compounds, Proc. Combust. Instit. 37 (2019) 1099-1105.

[12] K. O. Johansson, J. Zádor, P. Elvati, M. F. Campbell, P. E. Schrader, N. K. Richards-Henderson, K. R. Wilson, A. Violi, H. A. Michelsen, Critical Assessment of Photoionization Efficiency Measurements for Characterization of Soot-Precursor Species, J. Phys. Chem. A 121 (2017) $4475-4485$.

[13] M. Commodo, K. Kaiser, G. De Falco, P. Minutolo, F. Schulz, A. D'Anna, L. Gross, On the early stages of soot formation: Molecular structure elucidation by high-resolution atomic force microscopy, Combust. Flame 205 (2019) 154-164.

[14] J. W. Martin, D. Hou, A. Menon, L. Pascazio, J. Akroyd, X. You, M. Kraft, Reactivity of polycyclic aromatic hydrocarbon soot precursors: Implications of localized $\pi$-radicals on rim-based pentagonal rings, J. Phys. Chem. C 123 (2019) 26673-26682.

[15] A. Violi, Cyclodehydrogenation reactions to cyclopentafused polycyclic aromatic hydrocarbons, J. Phys. Chem. A 109 (2005) 7781-7787.

[16] R. Whitesides, M. Frenklach, Detailed kinetic Monte Carlo simulations of graphene-edge growth., J. Phys. Chem.. A 114 (2010) 689-703. 
[17] R. Whitesides, D. Domin, R. Salomón-Ferrer, W. A. Lester, M. Frenklach, Graphene layer growth chemistry: Five- and six-member ring flip reaction, J. Phys. Chem. A 112 (2008) 2125-2130.

[18] D. Hou, X. You, Reaction kinetics of hydrogen abstraction from polycyclic aromatic hydrocarbons by H atoms, Phys. Chem. Chem. Phys 19 (2017) 30772-30780.

[19] N. Mardirossian, M. Head-Gordon, How accurate are the minnesota density functionals for noncovalent interactions, isomerization energies, thermochemistry, and barrier heights involving molecules composed of main-group elements?, J. Chem. Theory Comput. 12 (2016) 4303-4325.

[20] M. Frisch, G. Trucks, H. Schlegel, G. Scuseria, M. Robb, J. Cheeseman, G. Scalmani, V. Barone, B. Mennucci, G. Petersson, et al., Gaussian 16, gaussian, Inc., Wallingford, CT (2016).

[21] M. Frenklach, Reaction mechanism of soot formation in flames, Phys. Chem. Chem. Phys 4 (2002) 2028-2037.

[22] D. S. N. Parker, R. I. Kaiser, T. P. Troy, M. Ahmed, Hydrogen abstraction/acetylene addition revealed, Angew. Chem. Int. Ed. 53 (2014) $7740-7744$.

[23] N. Hansen, M. Schenk, K. Moshammer, K. Kohse-Hoinghaus, Investigating repetitive reaction pathways for the formation of polycyclic aromatic hydrocarbons in combustion processes, Combustion and Flame 180 (2017) 250-261. 
[24] X. M. Cao, Z. R. Li, J. B. Wang, X. Y. Li, Rate rules for hydrogen abstraction reaction kinetics of polycyclic aromatic hydrocarbons and vinyl radical, Theoret. Chem. Acc. 139 (2020).

[25] Q. Mao, D. Hou, K. H. Luo, X. You, Dimerization of polycyclic aromatic hydrocarbon molecules and radicals under flame conditions, J. Phys. Chem. A 122 (2018) 87018708.

[26] A. Menon, J. W. Martin, G. Leon, D. Hou, L. Pascazio, X. You, M. Kraft, Reactive localized $\pi$-radicals on rim-based pentagonal rings: properties and concentration in flames, Proc. Combust. Inst. (2020) (accepted).

[27] K. Johansson, M. Head-Gordon, P. Schrader, K. Wilson, H. Michelsen, Resonance-stabilized hydrocarbon-radical chain reactions may explain soot inception and growth, Science 361 (2018) 997-1000.

[28] P. Elvati, A. Violi, Homo-dimerization of oxygenated polycyclic aromatic hydrocarbons under flame conditions, Fuel 222 (2018) 307-311.

[29] J. Happold, H. Grotheer, M. Aigner, H. Bockhorn, A. D'Anna, A. Sarofim, H. Wang, Combustion generated fine carbonaceous particles, Combustion Generated Fine Carbonaceous Particles, KIT Scientific Publishing (2009) 275-288.

[30] G. A. Kaminski, R. A. Friesner, J. Tirado-Rives, W. L. Jorgensen, Evaluation and reparametrization of the OPLS-AA force field for proteins via comparison with accurate quantum chemical calculations on peptides, J. Phys. Chem. B 105 (2001) 6474-6487. 
[31] M. J. Abraham, T. Murtola, R. Schulz, S. Páll, J. C. Smith, B. Hess, E. Lindahl, Gromacs: High performance molecular simulations through multi-level parallelism from laptops to supercomputers, SoftwareX 1 (2015) 19-25.

[32] M. Bonomi, D. Branduardi, G. Bussi, C. Camilloni, D. Provasi, D. Raiteri, D. Donadio, F. Marinelli, F. Pietrucci, R. A. Broglia, M. Parrinello, PLUMED: a portable plugin for free energy calculations with molecular dynamics, Comp. Phys. Comm. 180 (2009) 1961.

[33] S.-H. Chung, A. Violi, Peri-condensed aromatics with aliphatic chains as key intermediates for the nucleation of aromatic hydrocarbons, Proc. Combust. Instit. 33 (2011) 693-700.

[34] J. James, AMS2019-MOPAC: MOPAC Engine based on the MOPAC2016 source code, http://OpenMOPAC.net (2019).

[35] J. J. P. Stewart, Optimization of parameters for semiempirical methods VI: more modifications to the NDDO approximations and reoptimization of parameters, J. Mol. Model. 19 (2012) 1-32.

\section{Supplementary information}

The supplementary information for "Aromatic penta-linked hydrocarbons in soot nanoparticle formation" includes further details on the molecular dynamics simulations in the main paper. 